\title{
Beyond PDMS: off-stoichiometry thiol-ene (OSTE) based soft lithography for rapid prototyping of microfluidic devices $\dagger$
}

\author{
Carl Fredrik Carlborg, ${ }^{* a}$ Tommy Haraldsson, ${ }^{a}$ Kim Öberg, ${ }^{b}$ Michael Malkoch ${ }^{b}$ and Wouter van der Wijngaart ${ }^{a}$
}

Received 6th May 2011, Accepted 6th July 2011

DOI: $10.1039 / \mathrm{c} 1 \mathrm{lc20388f}$

\begin{abstract}
In this article we introduce a novel polymer platform based on off-stoichiometry thiol-enes (OSTEs), aiming to bridge the gap between research prototyping and commercial production of microfluidic devices. The polymers are based on the versatile UV-curable thiol-ene chemistry but takes advantage of off-stoichiometry ratios to enable important features for a prototyping system, such as one-step surface modifications, tuneable mechanical properties and leakage free sealing through direct UV-bonding. The platform exhibits many similarities with PDMS, such as rapid prototyping and uncomplicated processing but can at the same time mirror the mechanical and chemical properties of both PDMS as well as commercial grade thermoplastics. The OSTE-prepolymer can be cast using standard SU-8 on silicon masters and a table-top UV-lamp, the surface modifications are precisely grafted using a stencil mask and the bonding requires only a single UV-exposure. To illustrate the potential of the material we demonstrate key concepts important in microfluidic chip fabrication such as patterned surface modifications for hydrophobic stops, pneumatic valves using UV-lamination of stiff and rubbery materials as well as micromachining of chip-to-world connectors in the OSTE-materials.
\end{abstract}

\section{Introduction}

The field of microfluidics is a fast growing field with more than 10000 papers published on the topic over the last ten years, yet this has not been reflected in the number of commercial products based on microfluidics, which remains low. The vast majority of research in the field never reaches beyond a proof of concept prototype device and is seldom followed up by a product development. As pointed out by for example Mark et al. in their recent review of the field, ${ }^{1}$ one reason for this development gap between research and commercialization is the lack of a consistent material, fabrication and interfacing technology. ${ }^{2}$ Most of the conceptual microfluidic device development is still done using poly(dimethylsiloxane) (PDMS), which remains the material of choice for proof of concept prototyping since its introduction in the 1990s mainly because of its ease of use and attractive mechanical elastomeric properties. Unfortunately, for most applications a prototype conceptual device based on PDMS needs complete redevelopment to realize a commercial prototype. The reason is twofold, firstly, because the elastomeric properties are inadequate for many commercial products that

${ }^{a}$ Microsystem Technology, KTH Royal Institute of Technology, Osquldasväg 10, SE-10044 Stockholm, Sweden. E-mail: fredrik. carlborg@ee.kth.se; Tel: +4687907794

${ }^{b}$ Division of Coating Technology, Fibre and Polymer Technology, KTH Royal Institute of Technology, Teknikringen 56-58, SE-10044 Stockholm, Sweden

$\dagger$ Electronic supplementary information (ESI) available: NMR data for the three sample materials. See DOI: $10.1039 / \mathrm{c} 1 \mathrm{lc} 20388 \mathrm{f}$ need invariable surface chemistry and mechanical strength. Secondly, the PDMS fabrication processes are difficult to scale up and contain assembly steps, such as slow thermal curing or manual handling of thin elastomeric films that are incompatible with medium or large scale manufacturing.

To our knowledge there have been few, if any, attempts to develop a material specifically designed for the needs of lab-onchip applications. The availability of a prototyping strategy that can bridge the gap between research prototypes and commercial prototypes is very likely to increase the number of projects reaching a commercialization phase. A successful prototyping process requires a short turnaround time and a very high yield to allow for rapid redesign cycles. Furthermore, microfluidic devices need to resemble closely the final products in terms of surface chemistries and material properties. The latter is a complex requirement since commercial polymer microfluidic devices are either made from elastomeric polymers, such as PDMS in Fluidigm's Topaz chip, ${ }^{3}$ from hard plastics as in the disposable chips used in Biosite's Triage point of care system, ${ }^{4}$ or, more seldom, from a combination of elastomeric and hard plastics as in Abbot's I-STAT or Biacore's product. ${ }^{5,6}$

Hitherto, work to improve rapid prototyping of microfluidic devices has concentrated on new elastomeric formulations to replace PDMS, and on alternatives to thermoplastic prototyping. The work on new elastomer formulations aims at retaining the mechanical properties of PDMS while providing faster curing and improved solvent resistance. To this end, a UV curable elastomeric highly fluorinated perfluoropolyether polymer was developed that avoids the swelling of standard PDMS and that 
exhibits chemical resistance similar to that of Teflon ${ }^{\mathrm{TM}}$ and mechanical properties similar to those of PDMS. ${ }^{7}$ While solving some of the major problems of PDMS, other important issues, such as biocompatible layer bonding and surface patterning, remain unsolved.

The alternatives to PDMS prototyping either use off the shelf thermoplastics or specially designed UV-curable thermosets. The latter approach mimics commercial material properties, e.g. robustness and permanent surface modifications, to enable a seamless transition to high volume manufacturing. Thermoplastics are the natural choice for prototyping since the vast majority of commercial devices are made from medical grade variants of PMMA, polystyrene and cyclic olefin copolymer (COC). However, the use of thermoplastic materials in prototyping is hampered by expensive machines and tools for injection molding or hot embossing, complicated bonding of device layers and cumbersome and time consuming surface modification schemes. Hence, alternatives that require less development efforts and investment are highly desirable for prototype fabrication.

Compared to thermoplastics, thermosets are more suitable for rapid prototyping since they are shaped either by direct UV freeform lithography or by inexpensive replica molding, negating the need for expensive tools and machines. Freeform lithography is well suited for rapid prototyping, has great flexibility and rapid redesign turnaround, ${ }^{8,9}$ but requires excellent lithographic qualities of the polymer precursor and expensive lithography systems that are not realistic investments for most laboratories. On the other hand, replica molding of thermosets is a low-cost method, suitable for laboratories and if combined with a fast UV curing process the materials can also be used as a commercial material, e.g. in wafer-scale micromolding. ${ }^{10}$ A particularly promising thermoset utilizes UV-curable thiol-ene based materials. These are well suited for microfluidic devices thanks to the rapid turnaround enabled by a very high reaction rates, solvent resistance, and a low shrinkage stress, which prevents deformation of the thin geometries that are common in microfluidic devices. One report describes proof of concept microfluidic devices manufactured in commercial optical glue NOA81 (Norland Producs Inc, Cranbury, USA), which has mechanical properties similar to PMMA, superior light transparency $(>98 \%)$ and cures within seconds. ${ }^{11}$ Elastomeric thiol-enes have also been developed for nanoimprint lithography stamps, demonstrating excellent molding properties. ${ }^{11,12}$ While each of these examples shows the potential of thiol-ene system, they only addressed a limited set of current prototyping materials shortcomings.

To answer the challenge of bridging the gap between conceptual devices and commercial products, it is unlikely that a single material will suffice given the wide range of material properties currently found in commercial devices. Instead, research should be directed towards a material system where fully compatible elastomeric and stiff components can be used and combined as needed in a device. This would allow combining PDMS-like elastomeric materials, needed in e.g. valves, pumps or mixers, with stiffer materials for structural support, packaging and interfacing. Additionally, if such material systems would overcome the shortcomings of PDMS and enable stable surface modifications, good barrier properties to small molecules and/or a rapid curing process while retaining elastomeric properties, it would form an attractive alternative for the small number of currently available commercial devices that employ PDMS.
In this work, we introduce a family of UV-curable systems that addresses all of the above listed requirements for a microfluidic prototyping material system. By using the well-controlled chemistry and modularity of the thiol-ene system as a starting point, we have developed a novel platform of multi-functional and UV-curable off-stoichiometric thiol-ene (OSTE) polymers, specifically designed for microfluidics and lab-on-chip. Compared to previous thiol-ene systems, the OSTE polymers feature direct photo-patternable permanent surface modifications and low-temperature dry bonding. The materials and machining processes we use are inexpensive and closely resemble the well-accepted standard soft lithography process, which is important for a viable prototyping system.

In the first section of this paper, the advantages of thiol-ene polymers are described; in the second section we introduce the novel use of off-stoichiometry and the specific advantages it provide and in the third section we demonstrate the fabrication process and its main characteristics. Finally we apply the OSTE polymer to demonstrate microfluidic devices with direct bonding, controlled wetting, hydrophobic stops, capillary filling, pneumatic valves and the direct bonding and molding of OSTE fluidic connectors to the chips.

\section{Advantages of thiol-ene polymers in microfluidics}

The thiol-ene reaction has many properties in common with the popular photoinitiated thermoset (meth)acrylate systems that are extensively used in the coatings industry, such as spatial and temporal control of polymerization, solventless formulations, and good mechanical properties. It displays a rapid curing in the presence of little or no photoinitator, low volume shrinkage, delayed gelation point, little or no sensitivity to oxygen and low polymerization shrinkage stresses. Moreover, the thiol-ene reaction belongs to a family of reaction called "Click" reactions, denoting a set of powerful, very specific, and highly reliable "lock-and-key" reactions. ${ }^{13}$

Both the polymerisation characteristics and the material properties of thiol-ene systems make them very suitable for microfluidic device fabrication. The exceptionally low polymerization shrinkage stress allows thin device sections without warpage, the rapid polymerization reaction leads to device layer manufacturing times in the order of seconds and the availability of different multifunctional monomers allows for a large variety of mechanical and chemical properties. Furthermore, the specificity of the reaction and the unique alternating step-growth mechanism lead to well-defined homogeneous polymer networks that impart very useful material properties such as excellent optical clarity, a narrow glass transition and low permeability to common solvents. To understand the origins of these beneficial properties, we briefly describe the thiol-ene reaction mechanism. ${ }^{13,14}$

Thiol-ene photopolymerizations are step-growth radical polymerizations based on the addition of a thiol $(-\mathrm{SH})$ to a monomer with an ene-functional group (carbon double bond). The reaction, which is outlined in Fig. 1A, is normally triggered with a photoinitiator capable of forming radicals upon UV illumination (Initiation I), but is also triggered spontaneously with low wavelength UV-irradiation. ${ }^{15}$ The radical abstracts a hydrogen atom from the thiol to form a thiyl radical (Initiation 


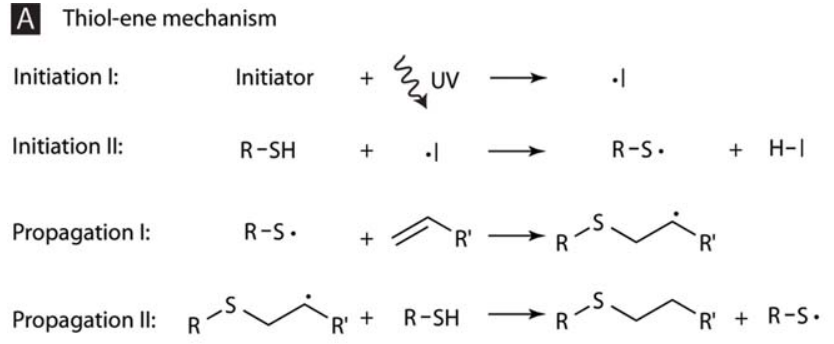

B Stoichiometry

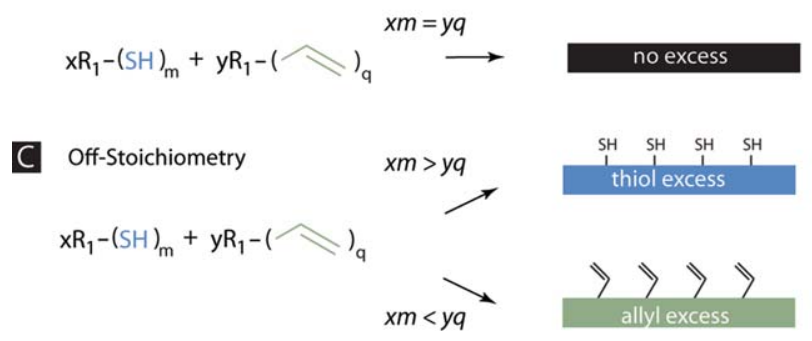

Monomers<smiles>C=CCn1c(=O)n(CC=C)c(=O)n(CC=C)c1=O</smiles><smiles>O=C(CCS)OCC(CO)(COC(=O)CCS)COC(=O)CCS</smiles>

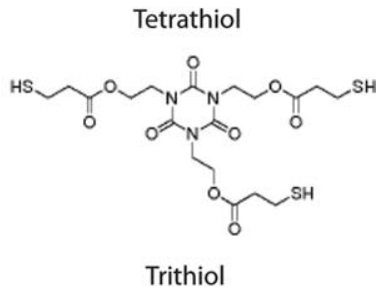

Fig. 1 (A) Reaction mechanism of UV-initiated radical thiol-ene coupling. (B) Standard stoichiometric formulations of thiol-ene systems lead to a complete polymerization and the absence of functional groups on the surface and in the bulk. (C) Off-stoichiometry formulations of thiol-enes allow for a well-defined amount of unreacted thiol or allyl anchors on the surface and in the bulk. (D) Monomers used for the OSTE-Thiol (90) and OSTEAllyl (30).

II) and the reaction propagates by a rapid cascade of polymerization reactions that, in a short time period, convert the liquid prepolymer into a solid polymeric material. First, the thiyl radical is added to an ene-functional monomer (Propagation I), next follows a chain transfer of the radical to a thiol functional group, regenerating a thiyl radical (propagation II). The chain transfer is the key step responsible for the final properties of the polymer since it imparts delayed gelation and a high ultimate conversion, thanks to decreased tendency of radicals to become trapped in the forming polymer network. Since most polymerization reactions reduce the monomer distance, any reactions occurring after the transformation from a liquid to a solid, i.e. the gel point, lead to shrinkage stress. Hence, the delayed gelation of thiol-enes greatly reduces the shrinkage stress experienced in the final material compared with other commonly used thermoset materials, e.g. acrylates, where the gel point is reached early on in the polymerization. ${ }^{16}$ The reason for the homogeneous network are twofold; firstly, the almost perfectly alternating reaction mechanism between thiols and enes that are sufficiently electronegative to prevent homopolymerization, e.g. vinyl ethers or allyl ethers, coupled with the rapid alternating reaction, leads to a polymer structure where crosslinks almost exclusively consist of identical sulfur and carbon bonds; and secondly the high conversion possible in most thiol-ene polymerizations reduces the presence of regions with a low crosslink density since almost all reactive groups are tied into the network. Consequently, irregular features such as dangling chain ends and regions with significantly higher crosslink densities are largely avoided.

\section{Novel use of off-stoichiometry in thiol-ene systems}

This section describes the novelty of the off-stoichiometry in thiol-ene (OSTE) polymers and the important advantages they provide as a rapid and flexible microfluidic device-manufacturing platform.

\section{Previous work}

A typical thiol-ene polymer is made out of two types of monomers, one with thiol functional groups, $x R_{1}-(\mathrm{SH})_{m}$, and the other with allyl functional groups, $y R_{2}-\left(\mathrm{CH}_{2}-\mathrm{CH}=\mathrm{CH}_{2}\right)_{n}$, where $y$ and $x$ are the number of monomers of each type and $n$ and $m$ are the number of functional groups on each. As with all previously demonstrated thiol-ene systems, one normally strives to maximize the mechanical strength, and the ultimate goal is to have an exact equal number of functional groups, $y n=x m$, with very few or no reactive groups remaining (Fig. 1B). The resulting polymer has a non-reactive surface that requires plasma activation to change the surface properties. Recent work, using thiol-ene glue for forming microfluidic stickers, describes surface inhibition during the polymerization process to create an unreacted sticky layer which allows for subsequent "glue bonding" to other substrates by an additional UV-curing step. ${ }^{11,12}$ Although useful for bonding, the method allows little control of the inhibited layer thickness, no discrimination between thiol and ene groups on the surface, and has no possibility of changing or tuning to softer mechanical properties.

\section{Novel off-stoichiometry}

In the OSTE polymers, we introduce off-stoichiometric formulations of thiol-enes with an excess of one of the functional groups, $x m \neq y n$, to achieve a polymer with remaining unreacted functional groups both in the bulk and on the surface (Fig. 1C). To avoid any homopolymerization between monomers with the same functional group and have a well-defined amount of unreacted thiols or enes, we use reactions between 
multifunctional thiol and allyl-ether monomers that are known to have a perfectly alternating polymerization mechanism and not homopolymerize. The novel use of intentional large offstoichiometric mixing ratios in thiol-enes leads to a number of advantages described below.

UV-patternable surface modifications. Many microfluidic devices rely on partial surface treatment to alter hydrophilicity or introduce bioconjugation molecules such as antibodies. Normally the surfaces of polymers first need to be activated, for example by breaking up covalent bonds with plasma activation, to create groups that can be used for further grafting. ${ }^{17}$ These radical-groups degrade over time and it is a challenge to get a dense and homogeneous surface coverage. In PDMS for example, stable surface treatment is difficult to achieve since the long and loosely cross-linked siloxane chains rotate and hide surface modifications after a few minutes to hours. ${ }^{18}$ The density of unreacted groups present on the surfaces of the OSTE polymers can be controlled by the degree of off-stoichiometry and is stable over time and evenly distributed over the entire surface. This makes them well suited for direct UV-initiated grafting of functional molecules using the specific thiol-ene "Click" reaction. ${ }^{19}$ In the Applications section, we show that with the application of a stencil mask, grafting can be precisely patterned on the surface.

Tuneable mechanical properties. A useful property for microfluidic devices is the ability to seamlessly combine stiff materials for the structural integrity with elastomeric materials used in active components such as pumps, mixers and valves. The thiolene chemistry provides a platform for building materials with different mechanical properties because of the wide availability of thiol and ene monomers. The additional advantage with the OSTE-polymers over standard thiol-ene polymers is that the mechanical properties can be tuned to the exact demands of the application by adjusting the off-stoichiometric ratio without changing monomer compositions. At off-stoichiometry, the monomers have fewer cross-links to the network, which in turn affects the stiffness and the glass transition temperature. In thiolenes, the glass transition occurs in a very narrow temperature span due to the homogeneous networks formed by the thiol-ene polymerization process. Consequently, by tuning the glass transition temperature slightly above room temperature by choosing the specific off-stoichiometry ratio, the mechanical properties of the OSTE-polymers can be temperature tuned in a narrow interval and rapidly transform from a glassy material (E-modulus in the GPa range) at room-temperature to a rubbery material (E-modulus in the MPa range) at temperatures only a few tens of degrees higher. As described below, this leads to important benefits in sealing and bonding.

Direct low temperature dry bonding. Most microfluidic chips require assembly of one or more microfluidic layers. There are numerous examples were pneumatic valves are needed to control the liquid flow, but where a rubbery material is ill suited for the entire cartridge. Such device manufacturing typically requires the integration of different types of materials, which introduces additional work in developing sealing and bonding techniques compatible with all materials. ${ }^{20}$ The bonding process of most polymers utilizes one of the following main strategies depending on the nature of the materials to be joined; thermal bonding, plasma activation, solvents or liquid glue. ${ }^{21}$ The main drawbacks of these methods with respect to microfluidics are excessive temperature requirements and/or the use of harsh reaction conditions. The latter can be exemplified by the plasma activation used for PDMS microfluidics, which is a destructive process that will affect any previous surface modifications. The thermal bonding and solvent bonding used for many thermoplastics are based on melting the polymers, which risks channel deformation and prevents surface functionalization of temperature sensitive molecules. UV-curable liquid glue is a gentle low-temperature process but requires an extra step in applying the glue and risks filling small structures such as channels.

In contrast, the OSTE polymers can directly use the reactive groups displayed on their surfaces to covalently link themselves to other surfaces, either directly or through a grafting step with a functional molecule. In this work we demonstrate direct lamination and bonding of two OSTE-polymers, with thiol and allyl excess respectively, through the UV-initiated thiol-ene reaction.

\section{Polymer formulations and properties}

In this section the composition and the characteristic properties of three example OSTE-polymers are discussed and compared with existing commercial materials.

\section{Materials and methods}

We developed three demonstrator OSTE materials to illustrate the versatility of the prototyping platform. All formulations are based on commercially, readily available and non-toxic monomers. ${ }^{22}$ The thiol and allyl monomers tris[2(3-mercaptopropionyloxy)ethyl]isocyanurate (trithiol), pentaerythritol tetrakis(2-mercaptoacetate) (tetrathiol) and triallyl-1,3,5triazine-2,4,6(1H,3H,5H)-trione (triallyl) were obtained from Sigma-Aldrich (Fig. 1D). The vinyl-functionalized polydimethylsiloxanes (AB109358, AB109359, AB109360, $\mathrm{AB} 112958)$ and the dimethylsiloxane copolymer (4-6\% (mercaptopropyl)methylsiloxane) (AB113728) were obtained from ABCR GmbH, Germany. To polymerize thick films, we used the photobleaching Lucirin TPO-L from BASF GmbH Germany as photoinitiator. The prepolymer formulations were mixed in predetermined off-stoichiometry ratios, degassed in a vacuum chamber and irradiated with a standard table-top UV-lamp (EFOS Lite, EFOS, Ontario, Canada) of $4 \mathrm{~mW} \mathrm{~cm}^{-2}$ equipped with a $365 \mathrm{~nm}$ wavelength band pass filter. We also mixed a series of different off-stoichiometric ratios of the same monomer system to demonstrate the mechanical tuning capability.

\section{Polymer characterization}

The mechanical properties, such as E-modulus and glass transition temperature were characterized using dynamic mechanical and thermal analysis (DMTA) (DMA Q800, PerkinElmer, Waltham, USA). The amount of thiol and allyl groups before and after polymerization was evaluated using FT-Raman spectrometry (Spectrum 2000, PerkinElmer, Waltham, USA) monitoring the peaks at $2575 \mathrm{~cm}^{-1}$ (thiol stretching vibration) and 
$1644 \mathrm{~cm}^{-1}$ (allyl stretching vibration). Spectra were acquired before and shortly after UV-polymerization. In the OSTEPDMS, the density of thiol and allyl groups per monomer is low and it was not possible to resolve the thiol and allyl peaks for these samples. The excess of reactive groups after polymerization motivates an investigation of the leaching of non-crosslinked material. This was accomplished by weighing the samples before and after extraction for $24 \mathrm{~h}$ in chloroform and subsequent drying in a vacuum oven at $50{ }^{\circ} \mathrm{C}$. Leaching with deuterated chloroform was also performed followed by a nuclear magnetic resonance analysis (NMR) to determine the composition of the extracted components. Because the common use of the polymers in a lab-on-chip setting is in aqueous solutions an identical leaching investigation was also performed using deionised water and deuterated water for the NMR analysis. For comparisons commercial grades of PDMS (Sylgard 184), PMMA (Plexiglas $\left.{ }^{\circledR}\right)$ and a thiol-ene based optical glue, NOA 81 (Norland Products), were also tested. NMR experiments were performed on a Bruker Avance $400 \mathrm{MHz}$ instrument. Proton NMR spectra were acquired with a spectral window of $20 \mathrm{ppm}$, an acquisition time of 4 seconds, and a relaxation delay of 1 second (see ESI $\dagger$ ).

In general the off-stoichiometry ratios affect both the mechanical and surface properties of the polymer. At higher offstoichiometry ratio, the glass transition temperature and the Emodulus at room temperature will be decreased. In Fig. 3A a thiol-ene system consisting of the tetrathiol and the triallyl monomers is mixed from $0 \%$ to $100 \%$ excess of the thiol functionality after polymerization. At room temperature the E-modulus goes from $1300 \mathrm{MPa}$ to $120 \mathrm{MPa}$ as the off-stoichiometric ratio is increased. At the same time the glass transition temperature drops from over $80{ }^{\circ} \mathrm{C}$ to $35^{\circ} \mathrm{C}$. An even larger range is accessible by switching the monomer structure, e.g. from a flexible pentaerythritol core to a stiff triazine ring. The number of active groups on the surface available for surface modification varies linearly with the degree of off-stoichiometry and the number of thiol or allyl groups per monomer. In this work we developed three sample materials that demonstrate the flexibility of the platform.

The first sample material, OSTE-Thiol (90) (Table 1), is an off-stoichiometry thiol-ene material composed of tetrathiol and triallyl monomers (Fig. 1D) with a large amount of excess thiolfunctionality $(90 \%)$. The resulting polymer network consists of fully reacted triallyls but to a large extent only partially reacted tetrathiols. The cross-linking density is thus lowered and the resulting material is a pliable, almost rubber-like material at room temperature, displaying a high density of thiol-groups on the surface. Close to the glass transition temperature at $35^{\circ} \mathrm{C}$ the material softens considerably, lowering the E-modulus from $250 \mathrm{MPa}$ (below $30{ }^{\circ} \mathrm{C}$ ) to only $10 \mathrm{MPa}$ (above $40{ }^{\circ} \mathrm{C}$ ) (Fig. $2 \mathrm{~B}$ ). The FT-Raman study confirms the presence of remaining thiol groups after complete polymerization (Fig. 3A left, filled area) and the absence of allyl groups, which indicates that the polymerization reaction is complete (Fig. 3A, right). The amount of extractable material in dichloromethane (DCM) amounted to $8 \%$, the extract consisting mostly of unreacted tetrathiol monomers but also impurities from the commercial monomers. The amount of extractables is nonetheless reasonably low considering the large excess of functionality present in the polymer. Moreover, in contrast to the long siloxane chains that bleed out from PDMS, ${ }^{23}$ these are low molecular weight monomers that have a low likelihood of depositing on the surface of the channels or interfering with sensors. We were not able to detect any extractable components in water. The OSTEThiol (90) material is used here as an easy to handle polymer for micromolding that seals well to other surfaces when heated to its $T_{\mathrm{g}}$.

The second sample material we produced, OSTE-Allyl (30), is composed of triallyl and trithiol monomers (Fig. 1D) with a $30 \%$ excess of allyl functional groups. To be able to produce a hard polymer with an excess of allyl groups, we replaced the flexible tetrathiol monomer with a stiffer heterocyclic trithiol monomer that has one less thiol group per molecule. The cross-linked network consists of fully reacted trithiol monomers and partially reacted triallyl monomers. The material is a hard and stiff polymer with an E-modulus of $1750 \mathrm{MPa}$ at room-temperature, similar to PMMA, that softens to approximately $10 \mathrm{MPa}$ when heated to its glass transition temperature at $68{ }^{\circ} \mathrm{C}$ (Fig. 2C). In Fig. 3B, the FT-Raman confirmed remaining unreacted allyl groups and the complete absence of thiol groups in the cured polymer. No extractable components were detected in water and, as expected, at a lower off-stoichiometry as compared to the OSTE-Thiol (90), the amount of extractable material in chloroform is lower. A detailed study of the extractable material in a ${ }^{1} \mathrm{H}-\mathrm{NMR}$ showed it consisted of allyl monomers that had not reacted with the network and impurities from the commercial monomers. We use the OSTE-Allyl (30) as a hard substrate material that can be covalently bonded to the OSTE-Thiol (90) materials using the UV-initiated thiol-ene "Click" reaction. The OSTE-Allyl (30) can be machined and drilled in without cracking, while at the same time be cast using standard micromolding. The dense network formed by the OSTE-polymer and the properties measured on stoichiometric thiol-enes with comparable backbones in previous work ${ }^{24}$ indicates that the gas permeability is much lower than for PDMS.

Table 1 Properties of the demonstrator OSTE-materials and reference materials

\begin{tabular}{|c|c|c|c|c|c|c|}
\hline Sample material & Monomers & $\begin{array}{l}\text { Functionality } \\
\text { mixing ratio }\end{array}$ & $\begin{array}{l}\text { E-modulus @ } \\
25^{\circ} \mathrm{C} / \mathrm{MPa}\end{array}$ & $T_{\mathrm{g}} /{ }^{\circ} \mathrm{C}$ & $\begin{array}{l}\text { Extractable in } \\
\text { chloroform }\end{array}$ & $\begin{array}{l}\text { Extractable } \\
\text { in water }\end{array}$ \\
\hline OSTE-Thiol (90) & Tetrathiol : triallyl & $1.9: 1$ & 250 & 35 & $8 \pm 2 \%$ & $0 \%$ \\
\hline OSTE-Allyl (30) & Triallyl : trithiol & $1.3: 1$ & 1740 & 68 & $2 \pm 1 \%$ & $0 \%$ \\
\hline OSTE-PDMS (20) & Thiol-PDMS : vinyl-PDMS & $1.2: 1$ & 0.33 & -36 & $10 \pm 2 \%$ & $0 \%$ \\
\hline References & & & & & & \\
\hline PDMS (Sylgard 184) & Vinyl siloxanes & $1: 1$ & 0.8 & -135 & $5 \pm 1 \%$ & $0 \%$ \\
\hline PMMA (Plexiglas®) & Methacrylates & $1: 1$ & 1800 & 105 & Dissolves & $0 \%$ \\
\hline NOA 81 (Norland Products) & Thiol and ene monomers & $1: 1$ & 1400 & 75 & $2 \% \pm 1 \%$ & $0 \%$ \\
\hline
\end{tabular}



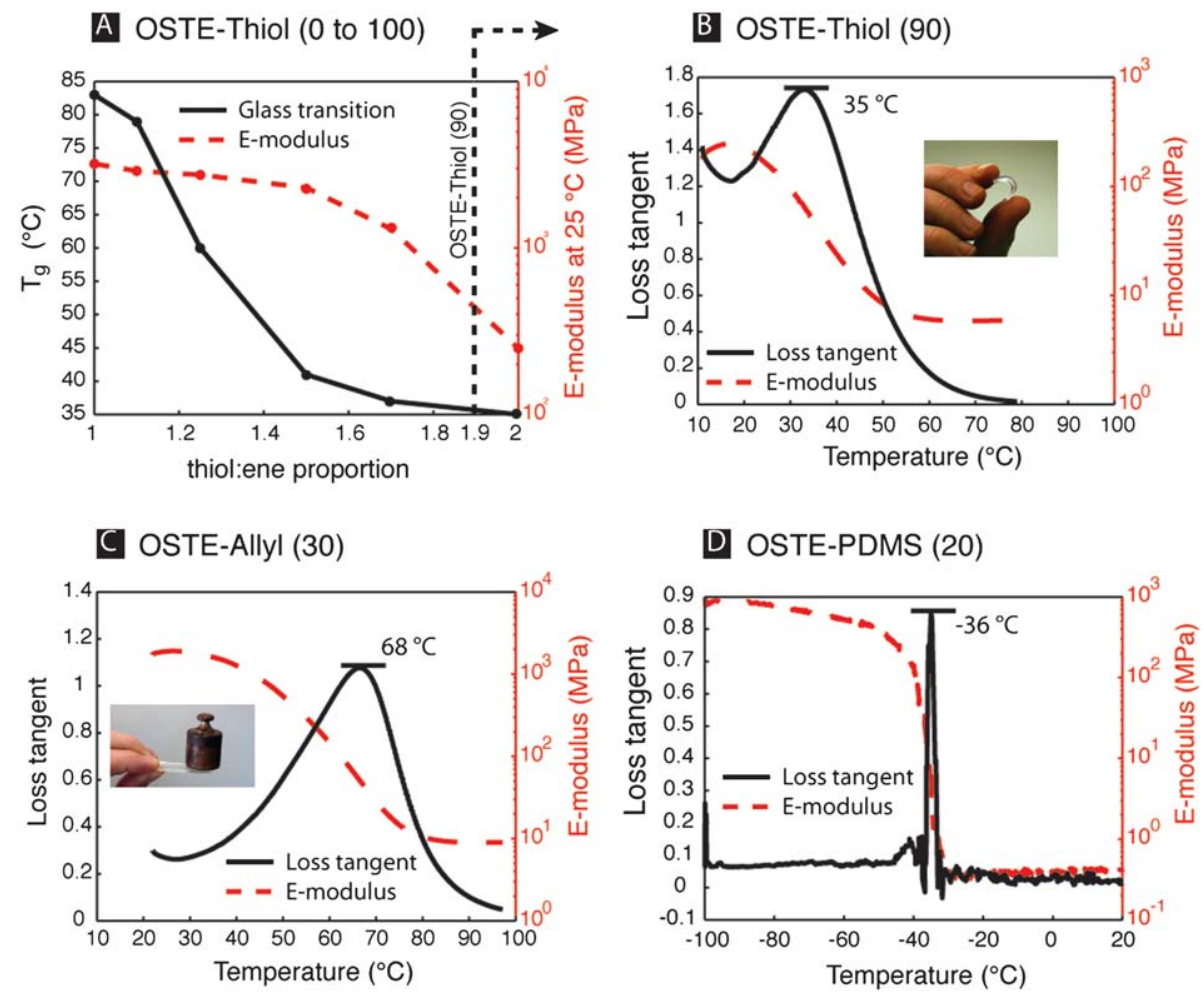

Fig. 2 Dynamic Mechanical and Thermal Analysis (DMTA) of the E-modulus and loss tangent of the three demonstrator materials. The peak of the loss tangent indicates when the glass transition temperature is reached. In (A) the OSTE-Thiol (90) with $90 \%$ excess of thiol groups is a medium stiff material $(250 \mathrm{MPa})$ at room temperature and below $\left(<30^{\circ} \mathrm{C}\right)$, but softens $(\sim 10 \mathrm{MPa})$ close to the biologically relevant temperature of $37^{\circ} \mathrm{C}$. In $(\mathrm{B})$ the much stiffer OSTE-Allyl (30) with 30\% excess allyl remains stiff (1200 MPa) up until $68^{\circ} \mathrm{C}$. Heating above the glass transition temperature significantly facilitates release from the mold or improves bonding. In (C) the elastomeric OSTE-PDMS (20) has a glass transition temperature at $-36{ }^{\circ} \mathrm{C}$ and an E-modulus of $350 \mathrm{kPa}$.

The final example material is used to demonstrate the flexibility of the thiol-ene platform. The OSTE-PDMS (20) is an elastomeric polymer with siloxane chains cross-linked using thiol-ene chemistry. The thiol-PDMS and vinyl-PDMS oligomers that are mixed together are of the same type as standard PDMS, where the only difference is the functional groups (thiol instead of silyl hydride). They are fully miscible and no phase separation occurs. The OSTE-PDMS (20) also displays high gas permeability and adsorption of small molecules due to the PDMS backbones. However, in contrast to PDMS, the 20\% excess of unreacted thiol groups enables surface modification using thiol-ene "Click" chemistry. The E-modulus of $350 \mathrm{kPa}$ at room temperature is lower than, but comparable to, that of Sylgard 184 (Fig. 2D). Although no extractable components were detected in water, the main drawback of this material is the higher amount of extractable components in DCM, attributed to long siloxane chains that failed to integrate with the network because of the off-stoichiometric ratio. Integrated and UVbonded together with the hard OSTE-Allyl (30) it is demonstrated for applications where valves or flexible membranes are needed as described below. The lifetime of the surfaces has not yet been investigated in detail but microfluidic OSTE-polymers were successfully surface modified one month after fabrication with no noticeable deterioration in performance. Further, there are no obvious mechanisms for deactivation of the surface since excess functionality is homogenously distributed throughout the polymer network; hence there is no concentration gradient that strives to deplete the surface functional groups. Furthermore, thiols and allyls are stable under ambient conditions.

\section{Device fabrication}

In this section we describe the fabrication steps of a complete microfluidic chip in the stiff OSTE-Allyl (30) and soft OSTEThiol (90). The process is similar to PDMS processing. Major differences are that the thermal curing step for PDMS is replaced by a rapid UV-curing for OSTE and that the bonding of layers does not require plasma treatment.

\section{Moulding process}

As mould masters we used silicon wafers with SU-8 patterns spin-coated with Teflon (Teflon AFTM 1600, DuPont, USA). After mixing and degassing, the prepolymer was poured on the mold (Fig. 4). The viscosity of the OSTE-Thiol (90) and OSTEAllyl (30) is lower than PDMS and degassing can be performed either by leaving the mixed prepolymer in a dark location for 1-2 hours or by actively degassing in a vacuum chamber for less than a minute. To planarize the top surface, we used thin polycarbonate plates with spacers to control the thickness of the polymer layer. The prepolymer was then exposed to UV-light (365 nm, $4 \mathrm{~mW} \mathrm{~cm}{ }^{-2}$ ) using a tabletop laboratory UV-lamp. 
OSTE-THIOL (90)
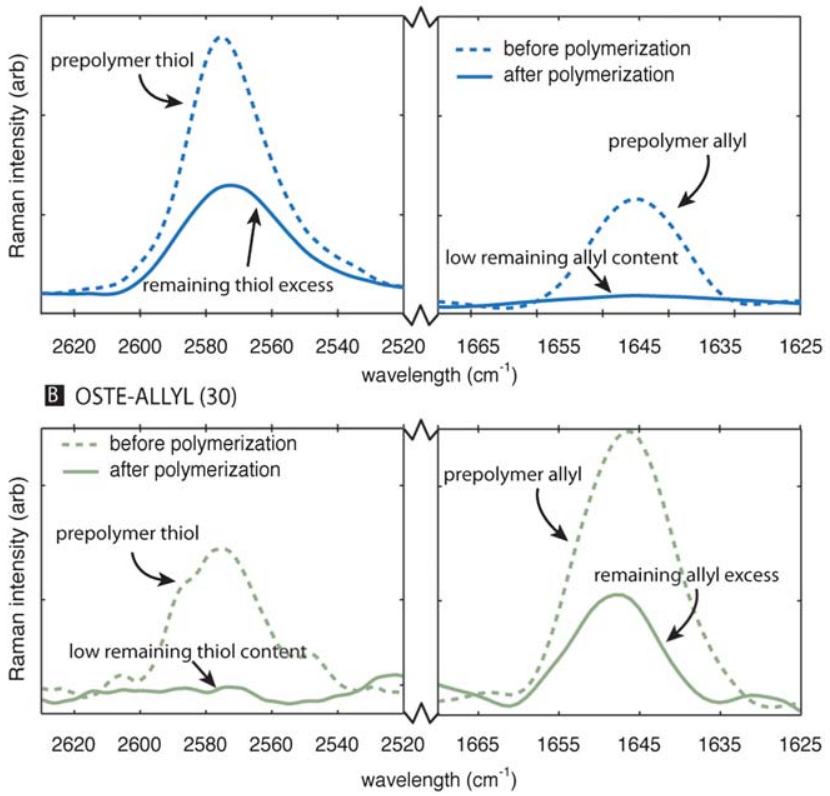

Fig. 3 Investigation of the remaining thiol and allyl groups in the OSTEThiol (90) (A) and OSTE-Allyl (30) (B) using FT-Raman spectroscopy. The two graphs on the left show the signal for the thiol group at $2575 \mathrm{~cm}^{-1}$ and the graphs on the right side show the signal for the allyl group at $1644 \mathrm{~cm}^{-1}$. The dotted lines represent the signal before polymerization and the solid lines/filled areas represent the remaining signal after complete polymerization. Before polymerization both samples (A) and (B) show the presence of both thiol and allyl groups, whereas after polymerization only thiol groups remain in the OSTE-Thiol (90) and only allyl groups remain in the OSTE-Allyl (30).

A $500 \mu \mathrm{m}$ thick film cures in approximately 30 seconds. The polymer film was then released from the mold and from the polycarbonate carrier. To facilitate the release of hard OSTEmaterials, such as the OSTE-Allyl (30), the mold was heated to the $T_{\mathrm{g}}$, at which the polymer softens and releases without damaging the mold. The OSTE-polymers are molded and the limiting factor in feature dimension is the resolution of the mold. Stoichiometric thiol-ene polymers with similar backbones have previously shown capability of nanometre resolution ${ }^{25}$

\section{Bonding process}

Two layers of OSTE polymers with different excess groups can be covalently bonded when exposed to UV-light. Since there is no initiator available in the already polymerized material, UV-light with a wavelength of $254 \mathrm{~nm}$ was used to initiate the thiol-ene polymerization reaction between the thiol and the allyl groups at the interface. Here, OSTE-Allyl (30) was bonded to OSTE-Thiol (90), Fig. 4C. The layers were aligned, brought into contact and heated to the $T_{\mathrm{g}}=35{ }^{\circ} \mathrm{C}$ of the OSTE-Thiol (90). At this temperature the polymer softens and conforms to micro- and nanoirregularities on the harder OSTE-Allyl (30) surface. Next the layers were exposed to UV-light (EFOS Lite, unfiltered medium pressure $\mathrm{Hg}$ spectrum). The exposure time varied from 5 min to $10 \mathrm{~min}$ because of the low light intensity at $254 \mathrm{~nm}$ wavelength produced by the medium pressure mercury lamp. The bond strength was tested by a blister test, in which pressurized air is pneumatically connected to a $5 \mathrm{~mm}$ diameter cavity between the bonded layers. When ramping the differential pressure, the layers withstood up to 4 bars, where after the bulk material ruptured, indicating a bond strength higher than the material integrity, and surpassing the needs of most microfluidic applications.

\section{Surface modification}

The OSTE-polymers all display varying densities of thiol groups (OSTE-Thiol (90) and OSTE-PDMS (20)) or allyl groups (OSTE-Allyl (30)) that can directly be used for grafting surface functionalizations using the thiol-ene "Click" reaction. Since the reaction is UV-initiated, the grafting can be patterned using, for example, a plastic stencil mask. First, grafting solutions are prepared in a solvent, e.g. water, toluene or iso-propanol, depending on the solubility of the grafting molecule. As an illustration, $5 \% \mathrm{w} / \mathrm{w}$ of the synthesized allyl-functionalized dye, Disperse red, was dissolved in toluene and $0.5 \% \mathrm{w} / \mathrm{w}$ ITX (Firstcure ${ }^{\circledR}$ ITX, Albemarle Corp., Baton Rouge, USA) photoinitiator was added. The grafting solution was applied on the surface (Fig. 5) of the OSTE-Thiol (90) polymer and to attach the red dye only on selected parts, a stencil mask was aligned and contacted with the liquid. During exposure to UV-light (365 nm, $4 \mathrm{~mW} \mathrm{~cm}^{-2}$ ) for 60 seconds, the allyl-groups on the red dye react with the free thiol-groups on the polymer surface to form a thioether bond. After exposure the stencil mask was removed and the polymer was thoroughly washed with toluene and blow dried with nitrogen gas. The procedure can be repeated if other molecules are to be grafted on other areas of the chip. Fig. 5 shows the result of the letters "KTH" patterned on the OSTEThiol (90) surface using a red dye functionalized with allyl.

\section{Applications}

The OSTE-polymers can be used to prototype chips with similar mechanical properties as thermoplastics or PDMS but the greatest advantage may lie in its potential for rapid direct surface modification. In this section we first show how to change the wettability of the surfaces to create a hydrophobic stop in a hydrophilic channel, then demonstrate functional components in the form of pneumatic valves and finally show how the OSTEpolymers can be used to create UV-bonded microfluidic chip-toworld connections.

\section{Modification of wetting}

We first demonstrate the ability to efficiently and permanently modify the water contact angle of the OSTE-surfaces. Solutions of the allyl or thiol functionalized grafting molecules were prepared in a solvent. As a hydrophilic modifier we synthesized end-functionalized PEG-allyls and PEG-thiols $\left(M_{\mathrm{w}} 5 \mathrm{kDa}\right)$, which were diluted as $10 \% \mathrm{w} / \mathrm{w}$ solutions in 2-propanol with $0.5 \%$ w/w ITX photoinitiator (Firstcure ${ }^{\circledR}$ ITX, Albemarle Corp., Baton Rouge, USA). As hydrophobic modifiers we utilized allyl functionalized PDMS (vinylmethylsiloxane homopolymer, linear, AB109397) and thiol functionalized PDMS (poly(mercaptopropyl)methylsiloxane, AB113729), both from ABCR $\mathrm{GmbH}$, Germany. The PDMS molecules were diluted as $10 \%$ w/w solutions in toluene with $0.5 \% \mathrm{w} / \mathrm{w}$ ITX photoinitator. 

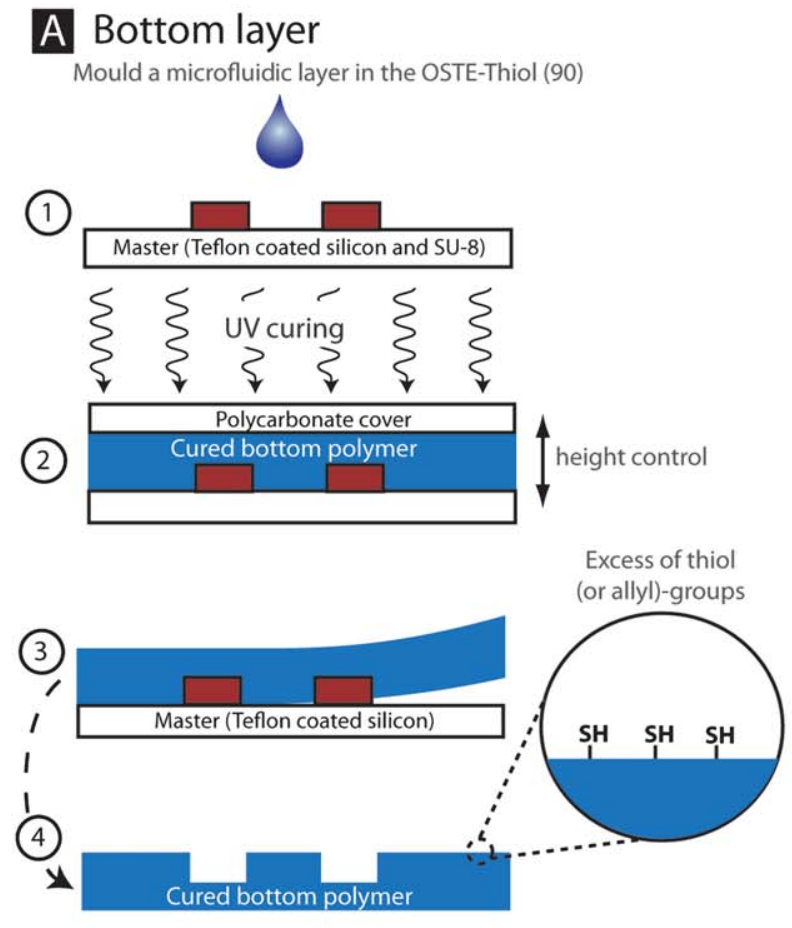

(3)

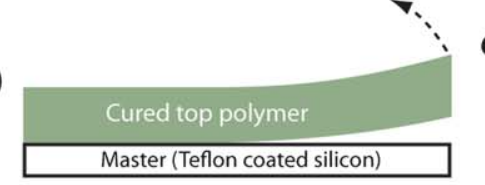

$60^{\circ} \mathrm{C}$

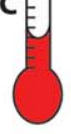

Heat to release from master. The polymer goes from glassy to rubber state.

(4)

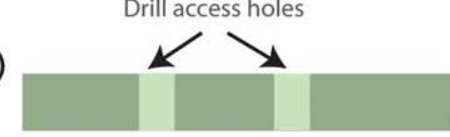

At room temperature (glassy state) the polymer is stiff and can be
C UV-bonding of layers

Lamination and UV-bonding of the OSTE-Thiol (90) and OSTE-Allyl (30) layers.

(1)

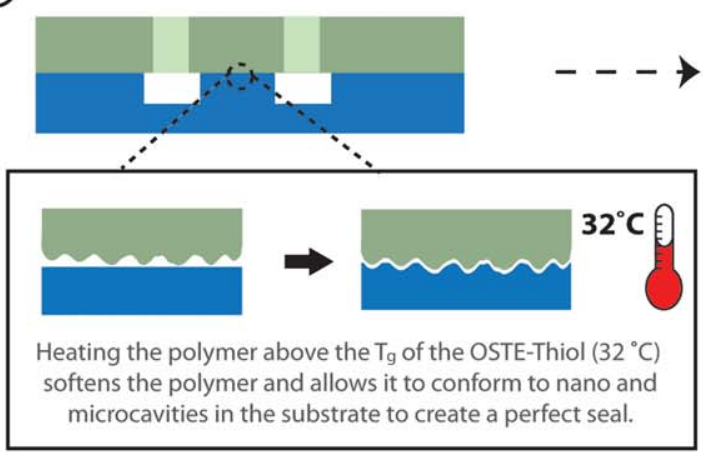
machined.
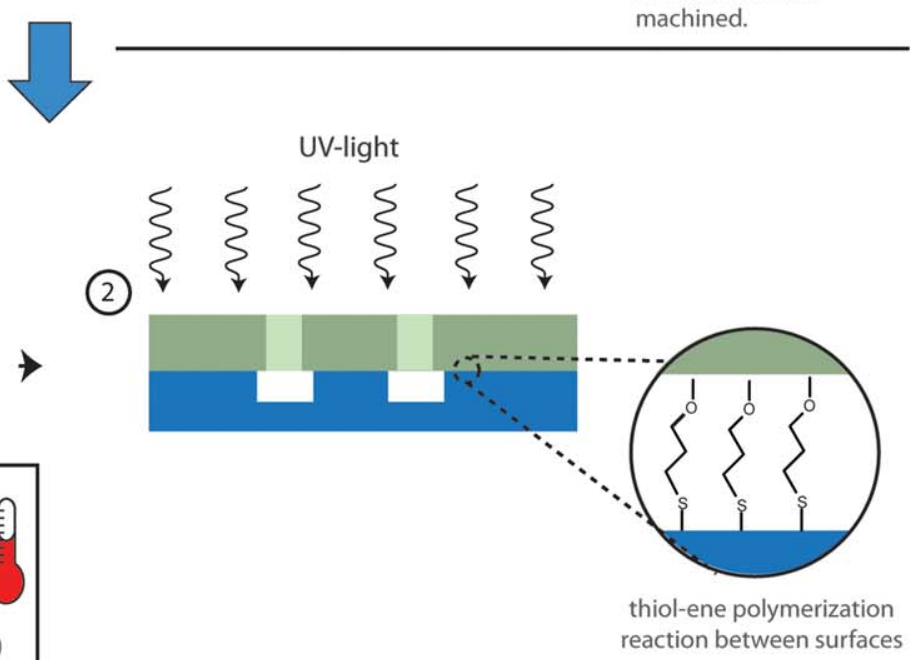

Fig. 4 The fabrication process of the OSTE materials is compatible with standard micromolding. (A) Here a bottom layer of OSTE-Thiol (90) is first molded. (B) A second stiffer layer of OSTE-Allyl (30) is also molded and access holes are drilled. (C) The stack is heated to the $T_{\mathrm{g}}$ of the OSTE-Thiol to allow the bond interface to conform to micro and nanoirregularities. Finally it is exposed to the UV-light to react with the thiols and the allyls to form a covalent bond.

To change the wetting of the OSTE-Thiol (90) and OSTE-PDMS surfaces, the solutions of allyl-functionalized molecules were used and to change the wetting of the OSTE-Allyl (30), the solutions of thiol-functionalized molecules were used. The surface modifiers were grafted on the different OSTE-surfaces following the above-described protocol.

Contact angles were measured using the sessile drop method. In Fig. 6 the different contact angles on modified and unmodified OSTE polymers are displayed. In their unmodified state, the OSTE-Thiol (90) and OSTE-Allyl (30) have contact angles of $76^{\circ}$ and $68^{\circ}$ respectively, similar to thermoplastics such as PMMA and PC. By grafting PEG or PDMS on the surface following the above described process, their wetting properties can be changed, Fig. 6A and B. The large change in contact angle indicates a high yield grafting reaction and an abundance of reactive sites on the surface. In contrast to the small multifunctional monomers used in the OSTE-Thiol and OSTE-Allyl materials, the OSTE-PDMS has only 5\% thiol groups along its siloxane chains, and the density of unreacted groups on the surface is therefore not high enough to have an impact on 


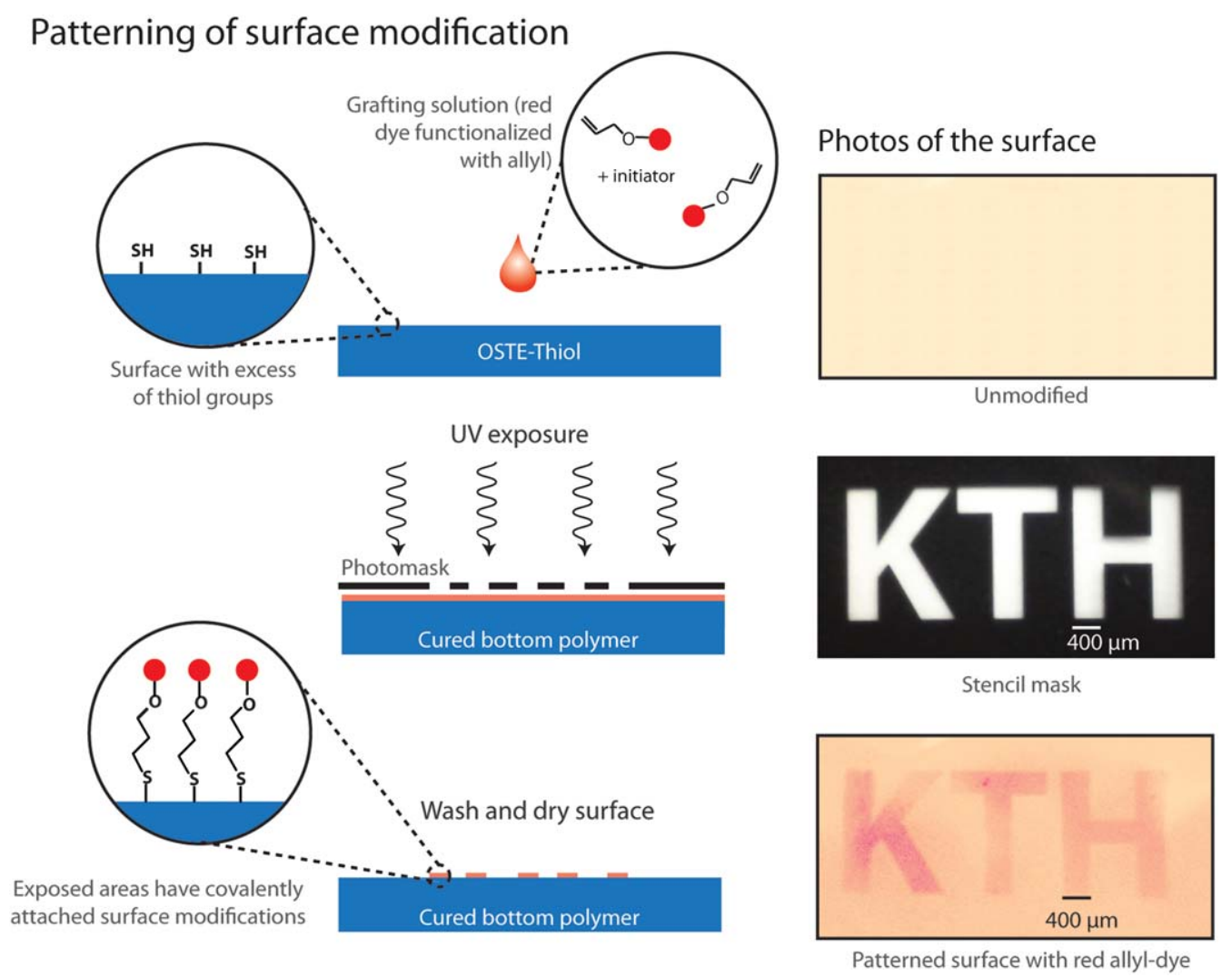

Fig. 5 Surface modification of the OSTE-surfaces through a one-step grafting process. Here the OSTE-Thiol (90) surface is patterned with an allyl functionalized red dye to form the letters "KTH". During UV exposure the allyl group on the dye molecule and the thiols on the surface react to form a thio-ether linkage. The OSTE-Thiol (90) is mixed with a $90 \%$ excess of thiol to allyl functional groups and the prepolymer is well stirred before polymerization. The bulk material and also the surface therefore have a homogeneous density of unreacted thiol groups. The slight difference in the surface coverage that can be observed in the patterned "KTH" is most likely because the surface was not saturated with the allyl-modified dye molecule due to difficulties in keeping the same concentration of dye molecules under the plastic film, which was manually applied.

macroscopic properties such as the contact angle. Instead a first layer of the allyl functionalized PDMS, mixed as above, is grafted to increase the number of reactive sites. These long PDMS chains react with one or more thiols on the surface and create molecular "spaghetti" of siloxane chains with several unreacted allyl groups. In a second step, the thiol functionalized PEG reacts with the allyls to create a PEG grafted surface. This procedure results in a clearly visible contact angle change (Fig. 6C).

\section{Hydrophobic stops}

Here we demonstrate hydrophobic retention valves by patterning hydrophobic molecules on the surface of two OSTE-polymers that are subsequently UV-bonded. We first manufactured a channel layer in the OSTE-Thiol (90) polymer (Fig. 7A(a)) with three straight channels, each $200 \mu \mathrm{m}$ wide and $30 \mu \mathrm{m}$ high and a flat bottom layer in the stiff OSTE-Allyl (30) (Fig. 7A(b)). By aligning a stencil mask, parts of the channel layer was patterned with allyl functionalized PDMS (vinylmethylsiloxane homopolymer, linear, $\mathrm{AB} 109397), \mathrm{CA}=97^{\circ}$. The same stencil mask was also aligned to the bottom layer which was modified with thiol functionalized PDMS (poly(mercaptopropyl)methylsiloxane, $\mathrm{AB} 113729), \mathrm{CA}=77^{\circ}$. Finally the layers were aligned, heated to $35^{\circ} \mathrm{C}$ to maximize the contact area between the layers, and exposed to unfiltered UV-light to initiate the covalent thiol-ene coupling (Fig. 7A(c)).

Water drops containing green, water based candy dye were placed on the drilled inlet holes. The liquid slowly filled all three channels by capillary action until it reached the hydrophobic areas, where it stopped (Fig. 7A(d)).

\section{Pneumatic valves}

The ability to produce pneumatic valves is important for a microfluidic prototyping material. A membrane of the elastomeric OSTE-PDMS was laminated between two crossing OSTEAllyl (30) channels with square cross-sections (width $=400 \mu \mathrm{m}$, height $=30 \mu \mathrm{m}$ ), heated to $35^{\circ} \mathrm{C}$ and exposed to unfiltered UVlight to covalently bond the layers (Fig. 7B). The top layer channel was used as liquid channels and the bottom layer channel as a pneumatic control channel. Applying a pneumatic pressure to the control channel closed the valves, which was observed through a microscope. As with PDMS pneumatic valves, the liquid channel must have a rounded cross-section to be able to completely close off the flow and a leakage of $5-10 \%$ was measured at liquid flow rates of $10 \mu \mathrm{L} \mathrm{min}{ }^{-1}$. The closing pressure was here defined to be when the leakage reached a minimum. Different closing pressures versus membrane thickness are plotted in Fig. 7B. The performance of the valves is 


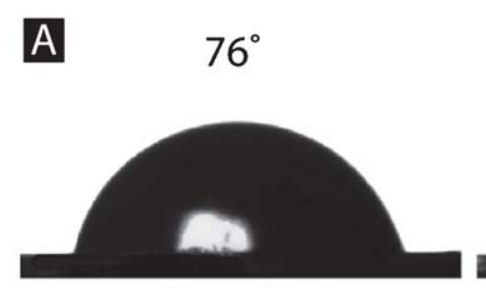

Unmodified

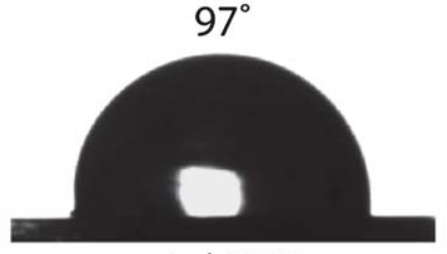

+ vinyl-PDMS

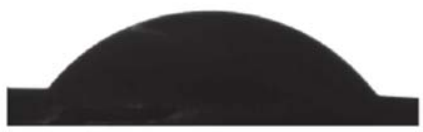

+ allyl-polyethylene glycol
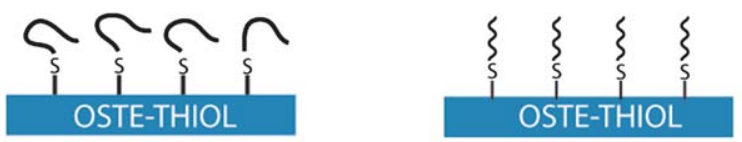

B

$68^{\circ}$

$77^{\circ}$

$35^{\circ}$

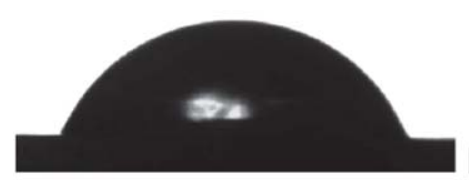

Unmodified OSTE-Allyl

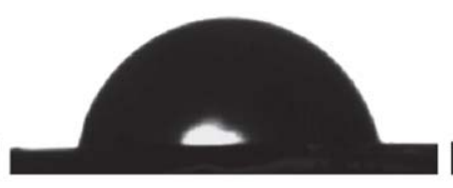

+ SH-PDMS

+ SH-polyethylene glycol
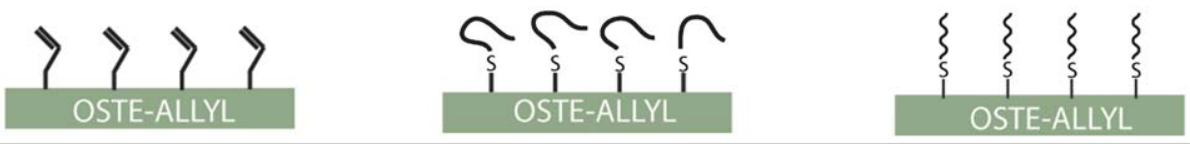

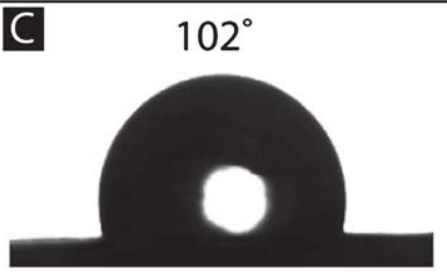

Unmodified

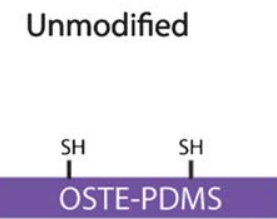

$86^{\circ}$

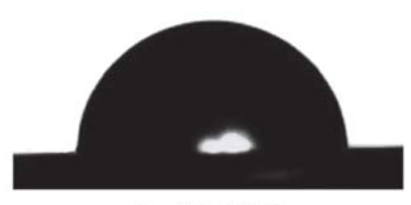

+ vinyl-PDMS

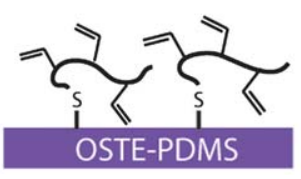

$49^{\circ}$

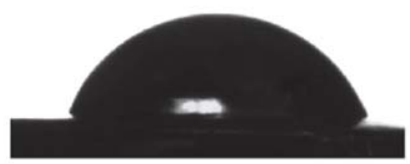

+ $\mathrm{SH}$-polyethylene glycol

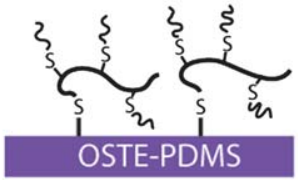

Fig. 6 The contact angles of the OSTE materials can be modified by direct UV-grafting. In (A) the OSTE-Thiol (90) has been grafted with vinylmethyl siloxane homopolymer (PDMS) to increase the contact angle and with allyl-PEG to lower the contact angle. (B) The OSTE-Allyl (30) has been modified with corresponding thiol functional molecules. The density of unreacted groups is lower in the OSTE-PDMS, and we first grafted long chains with vinylPDMS to inverse and multiply the functionality of each unreacted thiol. Finally the thiol functionalized PEG is grafted on the vinyl PDMS branches to significantly lower the contact angle.

comparable to that of Sylgard 184 PDMS valves ${ }^{26}$ but because of the slightly lower E-modulus the membrane thickness is not as critical.

\section{Rapid material integration: connectors}

Finally, we show a solution for integrated chip-to-world tube connectors. The ability of the OSTE-family of polymers to simplify the entire rapid prototyping cycle was demonstrated by machining fluidic connectors in the OSTE-Allyl (30) and drybond them to OSTE-Thiol (90) channel layers using UV-light under room temperature (Fig 7C). Fluidic ports based on the NanoPort ${ }^{\mathrm{TM}}$ were moulded in a PDMS mould master (Fig. 7C) and threaded to fit standard 6-32 coned fittings (UNC $3.5 \mathrm{~mm}$ ). The ports were aligned to the drilled inlet holes of the OSTEThiol (90), heated to $35^{\circ} \mathrm{C}$ to improve contact, and UV-bonded for 5 min with unfiltered UV (EFOS Lite, standard lamp without filter). The fittings were pressure tested to withstand air pressure exceeding 4 bars before rupturing.

\section{Scalability of the fabrication process; "bridging the gap"}

The typical OSTE-microfluidic chip production follows four steps; casting, surface grafting, bonding/sealing and dicing into individual chips. As demonstrated it is well suited as an alternative to the PDMS soft lithography and since the material properties can be tuned to mimic commercial thermoplastics, the process has potential to fill the gap between laboratory concepts and high volume series. In contrast to PDMS, the OSTE-process is also suitable for small scale commercial series production since the entire process from UV-casting to dicing can be performed on wafer scale, which increases manufacturing throughput and 
A Hydrophobic stops
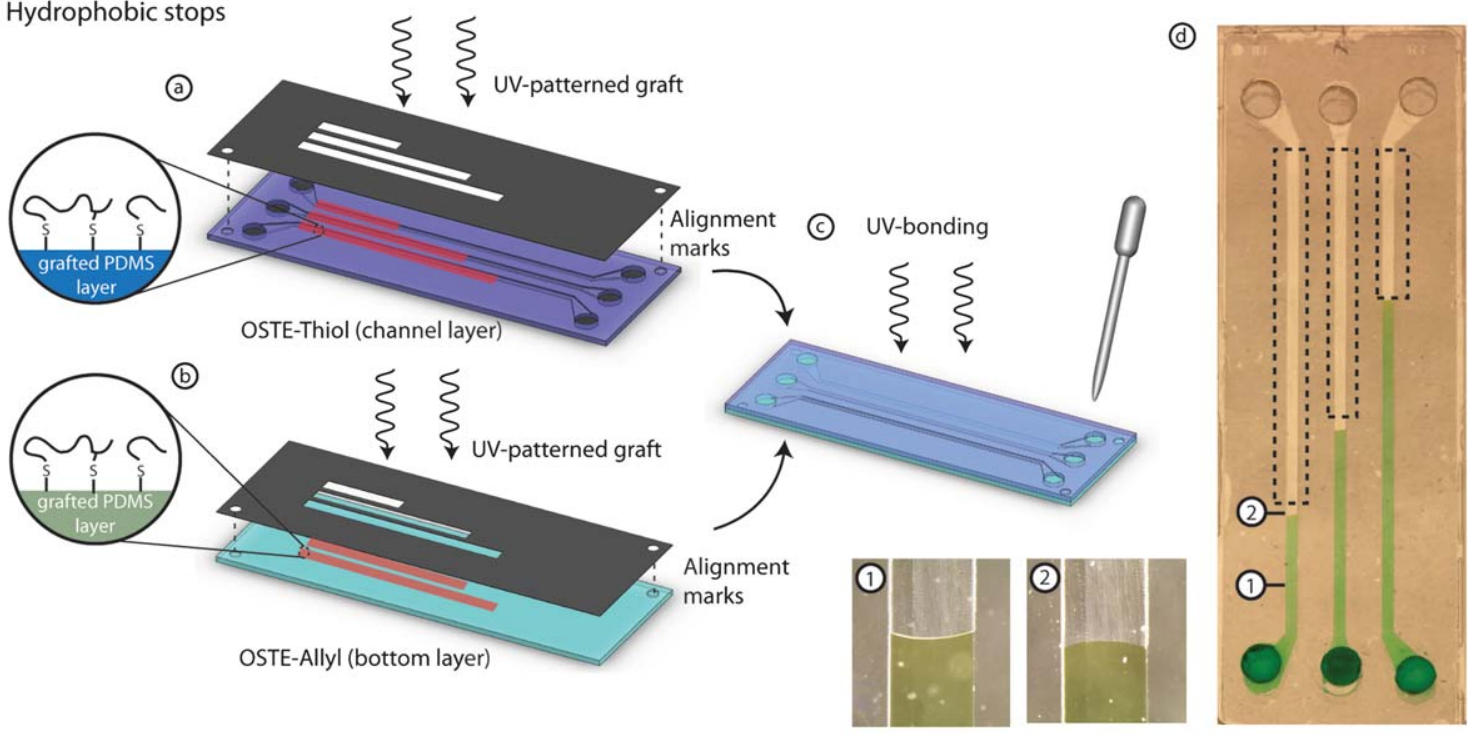

B Pneumatic valves

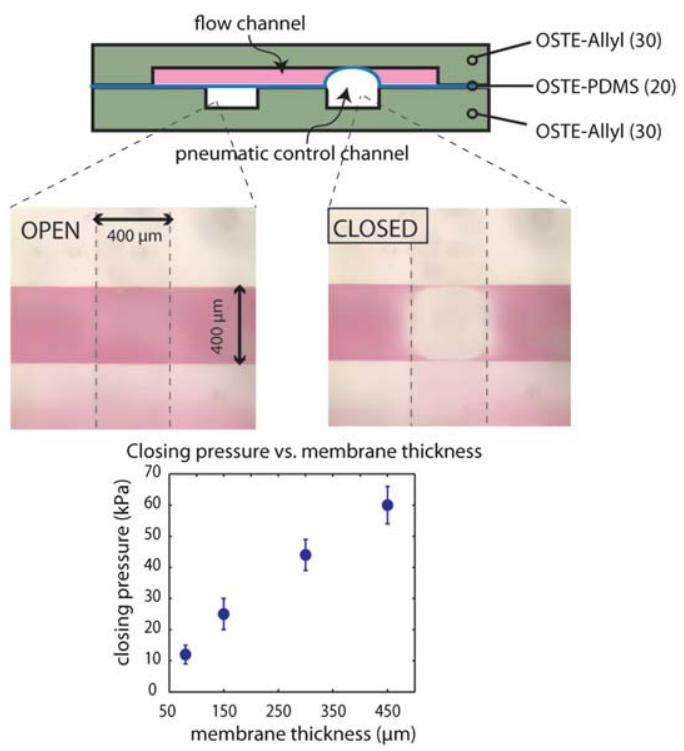

C

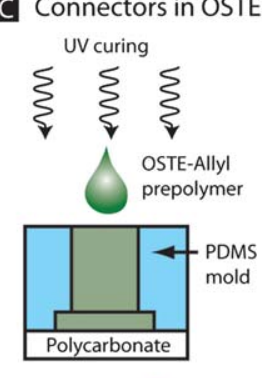

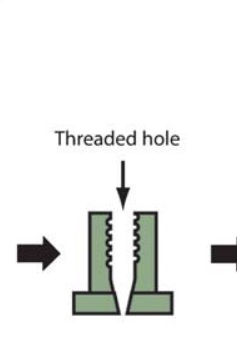

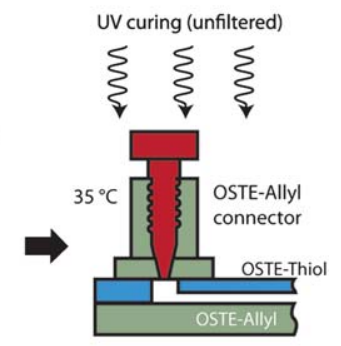

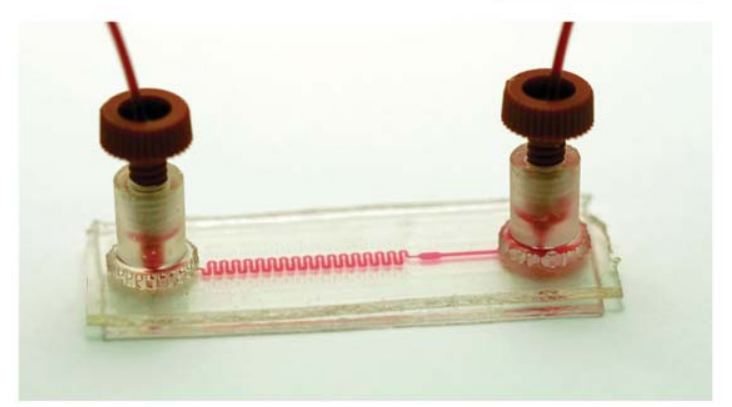

Fig. 7 (A) Hydrophobic retention valves created by patterning both the top and bottom channel layers with a hydrophobic coating (a and b) and then closing the chip by UV-bonding (c). Green coloured water filled the hydrophobic part of the channels but was held back at the patterned hydrophobic stops (d). Zoom-ins of the meniscus show the advancing contact angle in the hydrophilic part (1) and the stationary meniscus at the hydrophobic stop (2). (B) A pneumatic valve created by laminating and UV-bonding the elastomeric OSTE-PDMS between two layers of stiff OSTE-Allyl (30). The closing pressure for the valve versus membrane thickness is also plotted. (C) The OSTE-Allyl (30) is easily machined and can be moulded into connectors that are compatible with standard 6-32 coned nuts and can be directly UV-bonded to the OSTE-Thiol (90) microfluidic chips.

leads to costs far below that of small series thermoplastics molding.

\section{Conclusion}

In this article we have introduced a novel polymer platform, offstoichiometry thiol-enes (OSTE), aiming to bridge the gap between research prototyping and commercial production of microfluidic devices. The OSTE-platform is based on the thiolene system but instead of mixing the monomers in a $1: 1$ ratio, we introduced off-stoichiometric formulations. These are mixed with an excess of one of the functional groups to achieve a polymer with remaining unreacted functional groups both in the bulk and on the surface. The remaining functionality enabled both tunable mechanical properties by varying the off-stoichiometry ratio, patternable UV-grafting of functional molecules on the surface using thiol-ene "Click" reactions as well as onestep UV-bonding of OSTE-polymers. We showed that the platform can produce both glassy and elastomeric materials and also demonstrated an OSTE-PDMS material that has the advantages of OSTE but the well-known mechanical and chemical properties of PDMS. The sample OSTE polymers could all be cast and 
rapidly UV-cured (<30 s) on standard silicon/SU-8 molds. The wettability of the surface could be manipulated from hydrophilic $\left(<40^{\circ}\right)$ to hydrophobic $\left(>90^{\circ}\right)$ by UV-grafting of functional thiol or allyl molecules. Hydrophobic stops in a capillary filling microfluidic device were demonstrated by grafting patches of PDMS in the channels. Pneumatic valves were fabricated by direct UV-lamination of stiff channel layers with excess of allyl groups with an elastomeric membrane layer with excess of thiol groups. Finally the ability of the OSTE-family of polymers to simplify the entire rapid prototyping cycle was demonstrated by machining fluidic connectors in an OSTE material with allyl excess and dry-bond them to a microfluidic chip with thiol excess using UV-light under room temperature. We believe the OSTEpolymers have potential to bring more research to the commercialization by providing a rapid prototyping platform that mirrors thermoplastic properties but requires minimal investment and is compatible with existing micro-molding techniques used in many laboratories.

\section{Notes and references}

1 D. Mark, S. Haeberle, G. Roth, F. von Stetten and R. Zengerle, Microfluidic lab-on-a-chip platforms: requirements, characteristics and applications, Chem. Soc. Rev., 2010, 39, 1153.

2 H. Becker, Mind the gap!, Lab Chip, 2010, 10, 271.

3 Fluidigm, San Francisco, USA, www.fluidigm.com.

4 Biosite Inc., San Diego, USA, www.biosite.com.

5 Abbott Point of Care Inc., Princeton, USA, www.abbottpointofcare. com.

6 Biacore Life Science, Uppsala, Sweden, www.biacore.com.

7 J. P. Rolland, R. M. van Dam, D. A. Schorzman, S. R. Quake and J. M. DeSimone, Solvent-resistant photocurable "Liquid Teflon" for microfluidic device fabrication, J. Am. Chem. Soc., 2004, 126, 2322.

8 K. Haraldsson, J. Hutchison, R. Sebra, B. Good, K. Anseth and C. Bowman, 3D polymeric microfluidic device fabrication via contact liquid photolithographic polymerization (CLiPP), Sens. Actuators, B, 2006, 113, 454.

9 MicroTEC GmbH, Duisburg, Germany, www.microtec-d.com.

10 EVGroup GmbH, Florian, Austria, www.evgroup.com.

11 D. Bartolo, G. Degre, P. Nghe and V. Studer, Microfluidic stickers, Lab Chip, 2008, 8, 274.
12 M. Natali, S. Begolo, T. Carofiglio and G. Mistura, Rapid prototyping of multilayer thiolene microfluidic chips by photopolymerization and transfer lamination, Lab Chip, 2008, 8, 492.

13 C. E. Hoyle, T. Y. Lee and T. Roper, Thiol-enes: chemistry of the past with promise for the future, J. Polym. Sci., Part A: Polym. Chem., 2004, 42, 5301.

14 C. E. Hoyle and C. N. Bowman, Thiol-ene click chemistry, Angew. Chem., Int. Ed., 2010, 49, 1540.

15 N. B. Cramer, J. P. Scott and C. N. Bowman, Photopolymerizations of thiol-ene polymers without Photoinitiators, Macromolecules, 2002, 35, 5361 .

16 H. Lu, J. W. Stansbury and C. N. Bowman, Towards the elucidation of shrinkage stress development and relaxation in dental composites, Dent. Mater., 2004, 10, 979.

17 G. S. Ferguson, M. K. Chaudhury, H. A. Biebuyck and G. M. Whitesides, Monolayers on disordered substrates: selfassembly of alkyltrichlorosilanes on surface-modified polyethylene and poly(dimethylsiloxane), Macromolecules, 1993, 26, 5870.

18 C. Donzel, M. Geissler, A. Bernard, H. Wolf, B. Michel, J. Hilborn and E. Delamarche, Hydrophilic poly(dimethylsiloxane) stamps for microcontact printing, Adv. Mater., 2001, 13, 1164.

19 N. Gupta1, B. F. Lin, L. M. Campos, M. D. Dimitriou, S. T. Hikita, N. D. Treat, M. V. Tirrell, D. O. Clegg, E. J. Kramer and C. J. Hawker, A versatile approach to high-throughput microarrays using thiol-ene chemistry, Nat. Chem., 2010, 2, 138.

20 C. F. Carlborg, K. B. Gylfason, A. Kaźmierczak, F. Dortu, M. J. Bañuls Polo, A. Maquieira Catala, G. M. Kresbach, H. Sohlström, T. Moh, L. Vivien, J. Popplewell, G. Ronan, C. A. Barrios, G. Stemme and W. van der Wijngaart, A packaged optical slot-waveguide ring resonator sensor array for multiplex label-free assays in labs-on-chips, Lab Chip, 2010, 10, 281.

21 C.-W. Tsao and D. L. DeVoe, Bonding of thermoplastic polymer microfluidics, Microfluid. Nanofluid., 2009, 6, 1.

22 A. Nordberg, P. Antoni, M. I. Montanez, A. Hult, H. Von Holst and M. Malkoch, Highly adhesive phenolic compounds as interfacial primersfor bone fracture fixations, ACS Appl. Mater. Interfaces, 2010, 2, 654

23 S. Yunus, C. de Looringhe, C. Poleunis and A. Delcorte, Diffusion of oligomers from polydimethylsiloxane stamps in microcontact printing: surface analysis and possible application, Surf. Interface Anal., 2007, 39, 922.

24 L. Kwisnek, S. Nazarenko and C. E. Hoyle, Oxygen transport properties of thiol-ene networks, Macromolecules, 2009, 42, 7031.

25 E. C. Hagberg, M. Malkoch, Y. Ling, C. J. Hawker and K. R. Carter, Effects of modulus and surface chemistry of thiol-ene photopolymers in nanoimprinting, Nano Lett., 2007, 7, 233-237.

26 M. A. Unger, H.-P. Chou, T. Thorsen, A. Scherer and S. R. Quake, Monolithic fabricated valves and pumps by multilayer soft lithography, Science, 2000, 288, 113. 